\title{
HEAD-RELATED TRANSFER FUNCTION FILTER INTERPOLATION BY ROOT DISPLACEMENT
}

\author{
Hüseyin Hacıhabiboğlu, Banu Günel, Ahmet M. Kondoz. \\ Centre for Communication Systems Research (CCSR), \\ University of Surrey, Guildford, GU2 7XH, UK \\ \{h.hacihabiboglu, b.gunel, a.kondoz\}esurrey.ac.uk
}

\begin{abstract}
Head-related transfer function (HRTF) filters are used in virtual auditory displays for the binaural synthesis of the direction of a sound source over headphones. Once low-order HRTF filters are designed, the interpolation of these filters becomes an important issue for the synthesis of moving sound sources. An HRTF filter interpolation method based on the displacement of HRTF filter roots is proposed. It is possible to obtain a minimum-phase interpolated filter given that the original filters are also minimum-phase. The computational complexity of the method is the lower than that of the linear interpolation of magnitude responses.
\end{abstract}

\section{INTRODUCTION}

A head-related transfer function (HRTF) represents the spectral modification inflicted upon the sound wave by the acoustical transmission path from the sound source to the ear drum [1]. HRTFs are used in binaural virtual auditory displays for the synthesis of the direction of a sound source and the accompanying early reflections [2].

HRTFs can be obtained numerically with the help of geometrical models [3], or measured using human subjects or artificial mannequins $[4,5]$. Measurements are generally made for a limited number of positions on a spherical grid of points whose centre is occupied by a human subject or an artificial mannequin. If fulllength HRTFs obtained in these measurements are used, the filtering task involved in auralization becomes computationally too demanding. The general solution to this problem is to design and use low-order filters that are perceptually equivalent to using fulllength HRTFs.

If the limited number of measured HRTFs are used for auralization, smooth and continuous movement of sound sources cannot be achieved. Therefore, interpolation of HRTFs (or HRTF filters) for obtaining HRTFs (or HRTF filters) at intermediate directions is an essential step for the synthesis of virtual sound source motion and providing a seamless virtual auditory reality.

There exists a variety of methods applied to the HRTF interpolation problem. Most of the previously reported interpolation methods use the magnitude responses of minimum-phase reconstructed HRTFs rather than the filters designed from these HRTFs. These methods utilize linear [6], spline [7] or triangular [8] interpolation to obtain an interpolated magnitude response between the magnitude responses of two, three, or four HRTFs and then design a new interpolated HRTF filter from the obtained magnitude response.

This work was funded by EPSRC Research Grant GR/S72320/01
More complex HRTF interpolation approaches utilize spatial frequency response surfaces [9], non-linear interpolation with neural networks [10], active sensory tuning [11], approximation on a sphere using spherical basis functions [12], inter-positional transfer functions [13], and oversampling of DFT coefficients [14].

We distinguish HRTF filter interpolation from HRTF interpolation. The former is an interpolation between two (or more) filters modelling the HRTFs while the latter requires a filter to be designed after the interpolation process. The interpolation method proposed in this paper is carried out by displacing the filter roots since the frequency features which are essential cues in spatial hearing are represented more explicitly by the filter roots.

Section 2 summarizes the design of the HRTF filters used in the present work. HRTF filter interpolation using the zero-displacement measure is presented in Section 3. A brief comparison of linear interpolation and the proposed method is made and the conclusions are drawn in Section 4.

\section{HRTF FILTER DESIGN}

An HRTF can be represented as the combination of a minimumphase and an all-pass component. This property is frequently exploited in HRTF filter design. The HRTF is decomposed using the cepstrum analysis into a minimum-phase function, whose magnitude response is the same as that of the HRTF, and an all-pass function, whose phase represents the phase response of the HRTF. Apart from a small deviation for high frequencies, the phase response can be approximated to be linear (i.e. constant group-delay). Therefore, the interaural time delay (ITD) can be modelled as a constant delay. This constant delay can be obtained by finding the delay corresponding to the maximum of the cross-correlation of the original left and right ear HRTFs. Further, as there is no need to model the phase and magnitude response properties of the HRTF filter simultaneously, it is possible to design low-order filters [15].

The HRTF filters used as examples in this paper are obtained by minimum-phase reconstructing raw HRTFs and truncating the obtained minimum-phase impulse response to 65 samples by using a Hamming window centered at $t=0$. This operation provides the coefficients of $64^{\text {th }}$-order minimum-phase FIR filters. It should be be noted that the intepolation method proposed in this paper is applicable to all-poles IIR filters equally well. The HRTFs used in the design of the filters were obtained from the compact dataset of the MIT KEMAR HRTF database [5]. 


\section{INTERPOLATION OF HTRF FILTERS BY ROOT DISPLACEMENT}

Assume that we have two real-valued, $K^{\text {th }}$-order ( $K$ is even) FIR filters, $H_{1}(z)$ and $H_{2}(z)$ of the general form:

$$
H_{i}(z)=H_{i C}(z) \times H_{i R}(z),
$$

where $H_{i C}$, and $H_{i R}$ represent the complex and real filter sections such that:

$$
\begin{aligned}
& H_{i C}(z)=\prod_{k=1}^{N_{i}}\left[1-c_{i, k} z^{-1}\right]\left[1-c_{i, k}^{*} z^{-1}\right], \\
& H_{i R}(z)=\prod_{l=1}^{M_{i}}\left[1-r_{i, l} z^{-1}\right] .
\end{aligned}
$$

Here, $c_{i, k}$ and its complex conjugate $c_{i, k}^{*}$ are the complex zeros, and $r_{i, l}$ are the real zeros of the filter. The zeros of an FIR filter defining its characteristics around $\omega=0$ and $\omega=\pi$, and $0<$ $\omega<\pi$ can thus be separated.

Assume that $H_{1}(z)$ has $2 N_{1}$ complex zeros and $M_{1}=K-$ $2 N_{1}$ real zeros, and $H_{2}(z)$ has $2 N_{2}$ complex zeros and $M_{2}=$ $K-2 N_{2}$ real zeros. These filters can be represented in the $z$ domain as the real $\left(H_{1 R}(z)\right.$, and $\left.H_{2 R}(z)\right)$ and complex $\left(H_{1 C}(z)\right.$, and $\left.H_{2 C}(z)\right)$ sections. The aim of interpolation is to obtain new HRTF filter(s), $H_{\text {in }}(z)$, with magnitude responses between the two original filters.

Equations 1-3 represent a cascade form FIR filter [16]. The interpolation method proposed in this paper uses the roots that represent the cascaded second-order FIR sections for interpolation. In other words, corresponding filter roots are determined, and intermediate filter roots are derived from these. This requires the number of the complex zeros and the real zeros of the two filters to be equal (i.e. $N_{1}=N_{2}$ and $M_{1}=M_{2}$ ). Therefore, before the interpolation, the combination of real and complex zeros for each filter is equalized by replacing or substituting real zeros when necessary.

\subsection{Equalization of the Number of Real Zeros}

Three cases are possible for a given real-valued even-order FIR filter:

- Case 1: The filter has more than 2 real zeros $\left(M_{i}>2\right)$,

- Case 2: The filter has no real zeros $\left(M_{i}=0\right)$,

- Case 3: The filter has 2 real zeros only $\left(M_{i}=2\right)$.

Case 3 is selected as the baseline condition and the number of real zeros of the original filters are equalized to two such that the FIR filters can be represented as a combination of $K-2$ complex zeros and 2 real zeros.

For the baseline condition, the real zeros of the filters constitute second-order FIR sections, which have high-pass, low-pass or band-pass characteristics depending on the signs of the real zeros. The following two methods can be used for reducing or substituting real zeros for obtaining equal number of real zeros in both filters:

\subsubsection{Method 1}

For Case 1, the real zeros of the same sign are coupled and replaced with equivalent pseudo-complex zeros such that the number of the real zeros are decreased by two and the number of complex zeros are increased by two without significantly affecting the magnitude response.

Assume that we have a filter with two excess positive real zeros, $\left(r_{1}=a\right.$, and $\left.r_{2}=b\right)$ and we want to substitute a pair of pseudo-complex zeros $\left(c_{s}=p+j \epsilon\right.$ and $\left.c_{s}^{*}=p-j \epsilon\right)$ such that the error function,

$$
E(z)=\left(1-a z^{-1}\right)\left(1-b z^{-1}\right)-\left(1-c_{s} z^{-1}\right)\left(1-c_{s}^{*} z^{-1}\right),
$$

vanishes at $\omega=0$ (i.e. $z^{-1}=1$ ), where $0<a, b<1, a, b \in \mathbb{R}^{+}$, and $\epsilon$ is sufficiently small. When the error function is solved for $z=1, p$ can be found as:

$$
p \cong 1-\sqrt{(1-a-b+a b)}
$$

The derivation for negative real zeros is similarly done by substituting $z^{-1}=-1$.

If there are more than 2 real zeros, the method explained above is applied a number of times to obtain filters with 2 real zeros each. It must be noted that only the real zeros with the same sign can be replaced this way. This is the reason why the number of real zeros cannot be further reduced using the described method.

The method of substituting real zeros with equivalent pseudocomplex conjugate zeros does not violate the minimum-phase conditions since $\left|c_{s}\right|=\sqrt{p^{2}+\epsilon^{2}}<1$ for sufficiently small $\epsilon$, given that $0<a<1$ and $0<b<1$.

\subsubsection{Method 2}

For Case 2, the complex conjugate zeros that are the nearest to $z^{-1}=-1$ (i.e. $\omega=\pi$ ) are substituted with two real zeros such that the number of complex zeros are decreased by two while the number of real zeros are increased by two, without significantly affecting the magnitude response of the filter for lower frequencies.

Assume that we have a pair of complex conjugate zeros $(c=$ $-d+j g$, and $c^{*}=-d-j g$ ) and we want to substitute with a pair of real zeros $\left(r_{s 1}=r_{s 2}=-m\right)$ where $0<d, g, m<1$, and $d, g, m \in \mathbb{R}^{+}$such that the error, $E$ :

$$
E(z)=(2 d-2 m) z^{-1}+\left(d^{2}+g^{2}-m^{2}\right)
$$

is minimum. Given that $g$ is small for zeros near $\omega=\pi$, Equation 6 can be solved to give $m=-\Re\{c\}=d$.

The frequency spectrum at $\omega=\pi$ is well-defined. Therefore, even if negative real zeros do not exist, complex conjugate zeros are present at the immediate vicinity.

\subsection{Interpolation of the Complex Zeros}

The complex zeros of the original HRTF filters to be used in the interpolation are sorted with respect to their phases and paired according to their angular proximity. A zero displacement vector is calculated using the paired complex zeros as:

$$
\vec{\nu}_{k}=c_{1, k}-c_{2, k}, \quad k=1,2, \ldots, L,
$$

where $L=(K-2) / 2$. The interpolated complex zeros, $c_{i n t, k}$, can be calculated using this zero displacement vector as:

$$
c_{\text {int }, k}=c_{1, k}-\rho \vec{\nu}_{k}=[1-\rho] c_{1, k}+\rho c_{2, k},
$$




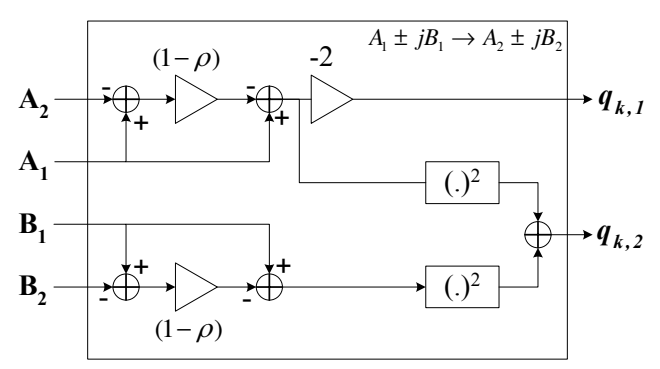

(a)
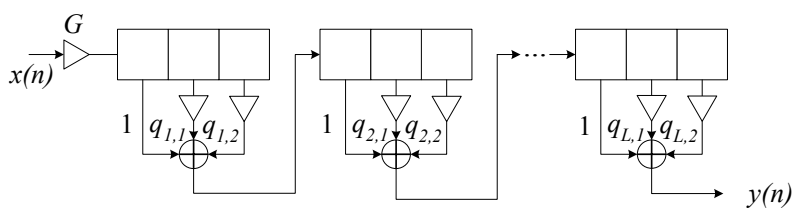

(b)

Figure 1: (a) Obtaining the cascaded FIR filter coefficients from original filter zeros, and (b) cascaded FIR filter blocks.

where $\rho$ is either a constant between 0 and 1 or a function of $k$ (i.e. $\rho=\rho(k))$. The magnitude of the interpolated zero is:

$$
\begin{aligned}
\left|c_{i n t, k}\right|= & \left|(1-\rho) c_{1, k}+\rho c_{2, k}\right| \\
= & {\left[(1-\rho)^{2}\left|c_{1, k}\right|^{2}+\rho^{2}\left|c_{2, k}\right|^{2}\right.} \\
& \left.+\left(2 \rho-2 \rho^{2}\right)\left|c_{1, k}\right|\left|c_{1, k}\right| \cos \xi\right]^{\frac{1}{2}},
\end{aligned}
$$

where $\xi$ is the angular separation between the zeros. Therefore, it can be shown for $0 \leq \rho \leq 1$ that the interpolated zeros are inside the unit circle (i.e. minimum-phase) if the original zeros are inside the unit circle (i.e. $\left|c_{1, k}\right|,\left|c_{2, k}\right|<1$ ).

Consider two zeros $c_{1, k}=A_{1}+j B_{1}$, and $c_{2, k}=A_{2}+$ $j B_{2}$ with their complex conjugate pairs. The interpolated complex conjugate zero pair, $\left\{c_{i n t, k}\right\}$ can be calculated as:

$$
\begin{aligned}
\left\{c_{\text {int }, k}\right\} & =\left[\rho A_{1}+(1-\rho) A_{2}\right] \pm j\left[\rho B_{1}+(1-\rho) B_{2}\right], \\
& =A_{\text {int }, k} \pm j B_{\text {int }, k}
\end{aligned}
$$

The interpolated pair of zeros constitute a second order FIR section, $H_{\text {int }, k}$, such that:

$$
\begin{aligned}
H_{i n t, k}(z) & =1-2 A_{i n t, k} z^{-1}+\left(A_{i n t, k}^{2}+B_{i n t, k}^{2}\right) z^{-2}, \\
& =1+q_{k, 1} z^{-1}+q_{k, 2} z^{-2} .
\end{aligned}
$$

The coefficients, $\left\{q_{k}\right\}$, of this second order block can thus be obtained easily from the real and complex parts of the interpolated zero (see Figure 1(a)). All of the obtained second-order FIR sections are cascaded as shown in Figure 1(b).

\subsection{Interpolation of the Real Zeros}

After the interpolation of the complex parts, the remaining two real zeros need to be interpolated. These zeros also constitute second order filter sections. They can be interpolated linearly using the polynomial form of coefficients:

$$
H_{i n t, R}(z)=\left[\rho \times H_{1 R}(z)\right]+\left[(1-\rho) \times H_{2 R}(z)\right],
$$
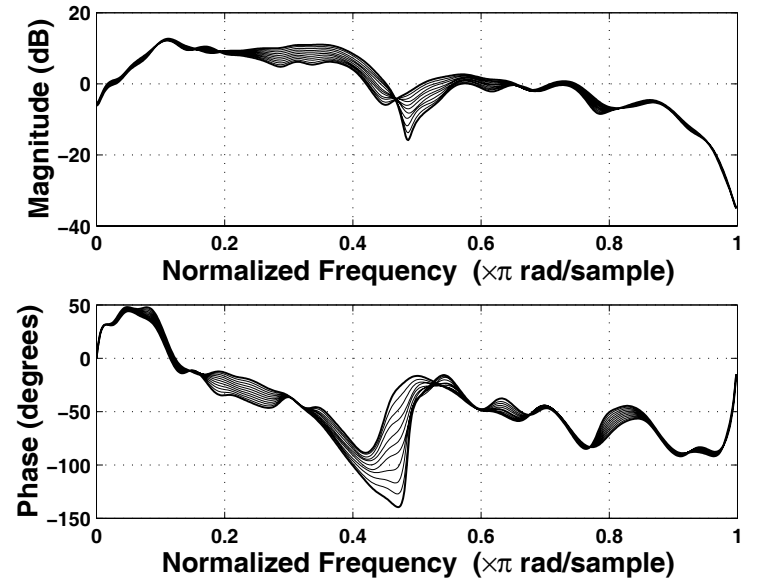

Figure 2: Interpolation between HRTF filters for $\theta=30^{\circ}, \phi=0^{\circ}$ and $\theta=40^{\circ}, \phi=10^{\circ}$.

where $0<\rho<1$. The filter $H_{\text {int }, R}(z)$ has a magnitude spectrum between the original filters $H_{1 R}(z)$ and $H_{2 R}(z)$.

\subsection{Combination of the Complex and Real Parts}

The interpolated filter is obtained by cascading the interpolated filter blocks for the complex and the real zeros. Therefore, the obtained filter is a cascade form FIR filter consisting of second order FIR sections that can be interpolated separately. Figure 2 shows the combined effect of interpolation applied to the complex and real parts of two HRTF filters. The interpolation parameter $\rho$ is varied from 0 to 1 with intervals of 0.1 . It may be observed that the proposed interpolation method is useful for interpolating between both the magnitude and the phase responses of the original filters. Figure 3(a) shows the interpolation of the HRTF filters on the horizontal plane. Figure 3(b) shows the interpolation of HRTF filters on the median plane. The darker regions signify higher values of magnitude spectra and the vertical spread of the associated region represents the significance of the given frequency feature.

\section{DISCUSSIONS AND CONCLUSIONS}

A new HRTF filter interpolation method for FIR filters based on the displacement of the filter roots was proposed in this paper. This method can be used for the interpolation of minimum-phase HRTF filters for the seamless synthesis of the three dimensional auditory space and sound source motion. The proposed method does not require explicit information of the shape of the filters' frequency spectra. Rather, it uses the zeros of the original filters in the interpolation stage. It guarantees that the interpolated FIR filter is minimum-phase.

In comparison with the linear interpolation of HRTF magnitude responses, the major advantage of the proposed method is its ability to interpolate between two magnitude spectra without having to redesign a filter. The calculation of the interpolated coefficients of cascaded FIR sections has a computational complexity of $O(n)$. The computational complexity of linear interpolation of magnitude spectra is $O\left(n \log _{2} n\right)$, because of the FFT operations involved. This makes the proposed method suitable and favourable for use in HRTF filter interpolation in real-time. 


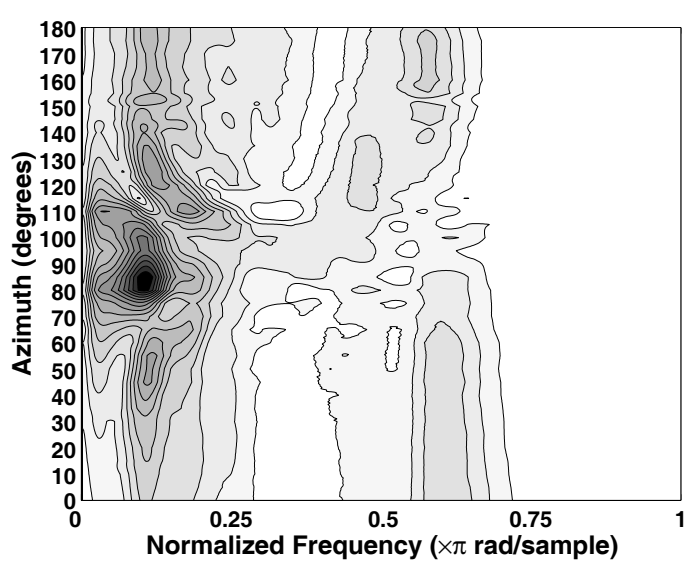

(a)

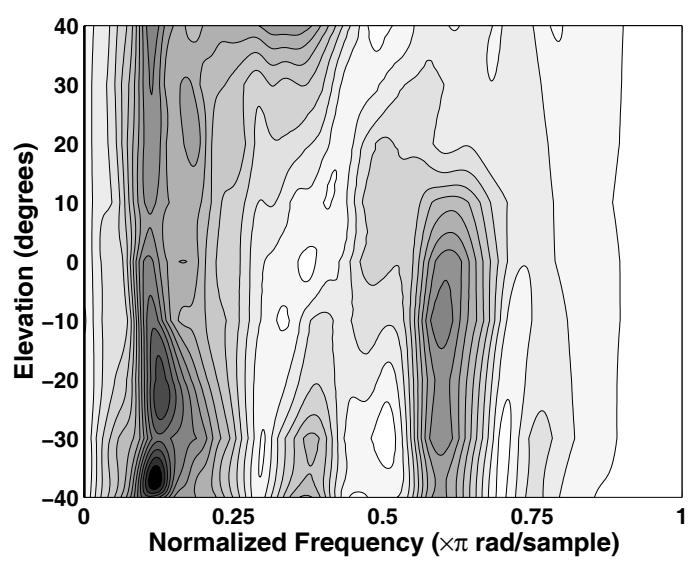

(b)

Figure 3: Interpolation using the zero displacement vector method (a) on the horizontal plane HRTFs, and (b) on the median plane HRTFs

Interpolation parameter can be selected differently for each corresponding root pair in the original filters. In this way, it would be possible to modify the magnitude spectrum of the interpolated filter nonlinearly. This would be useful in perceptually-based interpolation of the HRTF filters with the use of information on perceptually prominent spectral cues (see for example [17]). The proposed method can also be applied to all-poles IIR filters.

\section{REFERENCES}

[1] J. Blauert, Spatial Hearing: The Psychophysics of Human Sound Localization. Cambridge, MA, USA: MIT Press, 1997.

[2] M. Kleiner, B.-I. Dälenback, and P. Svensson, "Auralization - an overview," J. Audio Eng. Soc., vol. 41, no. 11, pp. 861875, 1993.

[3] C. P. Brown and R. O. Duda, "A structural model for binaural sound synthesis," IEEE Trans. Speech Audio Processing, vol. 6, no. 5, pp. 476-488, 1998.
[4] V. R. Algazi, R. O. Duda, D. M. Thompson, and C. Avendano, "The CIPIC HRTF database," in Proc. of 2001 IEEE Workshop on Applications of Signal Processing to Audio and Acoustics, New Paltz, NY, USA, Oct 2001.

[5] W. G. Gardner and K. D. Martin, "HRTF measurements of a KEMAR,” J. Acoust. Soc. Am., vol. 97, no. 6, pp. 3907-3908, 1995.

[6] E. Wenzel and S. H. Foster, "Perceptual consequences of interpolating head-related transfer functions during spatial synthesis," in Proc. of 1993 IEEE Workshop on Applications of Signal Processing to Audio and Acoustics, New Paltz, NY, USA, 1993, pp. 102-105.

[7] T. Nishino, S. Kajita, K. Takeda, and F. Itakura, "Interpolating head-related transfer functions in the median plane," in Proc. of 1999 IEEE Workshop on Applications of Signal Processing to Audio and Acoustics, New Paltz, NY, USA, 1999, pp. 167-170.

[8] L. Tronchin, V. Tarabusi, A. Farina, and A. Guisto, "Spatialization and acoustical simulation in the binaural technology," in Proc. of the International Symposium on Musical Acoustics, Perugia, Italy, 2001, pp. 461-464.

[9] C. I. Cheng and G. H. Wakefield, "Moving sound source synthesis for binaural electroacoustic music using interpolated head-related transfer functions (HRTFs)," Comput. Music J., vol. 25, no. 4, pp. 57-80, 2001.

[10] T. Nishino, M. Ikeda, K. Takeda, and F. Itakura, "Interpolating head related transfer functions," in Proc. of $7^{\text {th }}$ Western Pacific Regional Acoustics Conference, Kumamoto, Japan, 2000, pp. 293-296.

[11] P. R. Runkle, M. A. Blommer, and G. H. Wakefield, "A comparison of head-related transfer function interpolation methods," in Proc. of 1995 IEEE Workshop on Applications of Signal Processing to Audio and Acoustics, New Paltz, NY, USA, 1995, pp. 88-91.

[12] R. L. Jenison, M. F. Neelon, R. A. Reale, and J. F. Brugge, "Synthesis of virtual motion in 3D auditory space," in Proc. of $20^{\text {th }}$ Annual International Conference of the IEEE Engineering in Medicine and Biology, 1998, pp. 1096-1100.

[13] F. P. Freeland, L. W. P. Biscainho, and P. S. R. Diniz, "Interpositional transfer function for 3D-sound generation," $J$. Audio Eng. Soc., vol. 52, no. 9, pp. 915-930, 2004.

[14] M. Matsumoto, S. Yamanaka, M. Tohyama, and H. Nomura, "Effect of arrival time correction on the accuracy of binaural impulse response interpolation," J. Audio Eng. Soc., vol. 52, no. 1, pp. 56-61, 2004.

[15] D. J. Kistler and F. L. Wightman, "A model of head-related transfer functions based on principal components analysis and minimum-phase reconstruction," J. Acoust. Soc. Am., vol. 91, no. 3, pp. 1637-1647, 1992.

[16] A. V. Oppenheim and R. W. Schafer, Discrete-Time Signal Processing. Englewood Cliffs, NJ, USA: Prentice Hall, 1989.

[17] E. H. A. Langendijk, "Spectral Cues of Spatial Hearing," Ph.D. dissertation, Technische Universiteit Delft, Delft, the Netherlands, 2002. 